\title{
Prescribing of anticancer drugs in renal impairment: why can't we do better?
}

\author{
Emaad Abdel-Kahaar ${ }^{1} \cdot$ Oliver Zolk ${ }^{1}$ (D
}

Received: 14 December 2017 / Accepted: 21 December 2017 / Published online: 6 January 2018

(C) Springer-Verlag GmbH Germany, part of Springer Nature 2018

The number of patients with chronic kidney disease is increasing worldwide, and recent estimates indicate that chronic kidney disease is more common than diabetes (GBD 2016 Disease and Injury Incidence and Prevalence Collaborators 2017; Hill et al. 2016). The global prevalence of chronic kidney disease is currently between 11 and 13\% (Hill et al. 2016). The prevalence of renal insufficiency among cancer patients is even higher; every other cancer patient has a reduced glomerular filtration rate (GFR $<90 \mathrm{ml} / \mathrm{min})$, and 12 to $26 \%$ have a stage 3 and 4 kidney disease (i.e., GFR $<60 \mathrm{~mL} / \mathrm{min}$ ) (Launay-Vacher et al. 2016). Renal dysfunction in cancer patients can arise from a pre-existing kidney disease, can be related to the tumor (e.g., direct infiltration, compression, or paraneoplastic mechanisms), or can develop because of the nephrotoxic effect of cytotoxic agents.

The kidneys represent a major organ for the elimination of several anticancer drugs; thus, impaired renal function can alter pharmacokinetics and, consequently, systemic drug exposure. This can increase the risk of the toxic effects of anticancer drugs, which generally have a narrow therapeutic index. A relatively new and less established concept is that the kinetics of drugs eliminated by non-renal pathways can also be altered by renal insufficiency. Such indirect or secondary effects have been attributed to elevated uremic toxins and inflammation, modifying, e.g., hepatic transporter function and cytochrome P450 enzyme activity (Velenosi and Urquhart 2014). In this respect, it is imperative that oncologists are aware of the impact of renal function in terms of total drug clearance and the requirements of dose adjustments to avoid higher degree toxicities. However, dose adjustments of

Oliver Zolk

oliver.zolk@uni-ulm.de

1 Institute of Pharmacology of Natural Products and Clinical Pharmacology, University Hospital Ulm, Helmholtzstr. 20, 89081 Ulm, Germany antineoplastic treatments should not result in undertreatment and, therefore, in lower chances of cure.

Which exact dose should be used to account for the changes in the pharmacokinetics and pharmacodynamics that occur in renal dysfunction? According to the KDIGO (Kidney Disease: Improving Global Outcomes) clinical practice recommendation, physicians should adjust drug dosages primarily according to the approved product labeling; where there is no information in the product label, peer-reviewed literature recommendations should be used (Matzke et al. 2011).

In the article that accompanies this editorial, Grafe et al. 2018 compare the dosing recommendations provided in the official product labeling (summary of product characteristics, SmPC) with real-world prescribing practices for anticancer drugs in a German oncology center (Grafe et al. 2018). The authors evaluated the prescriptions of three commonly used cytostatic agents, which are substantially eliminated renally, namely cisplatin, ifosfamide, and etoposide. Nearly no drug prescriptions for patients with moderate or severe renal impairment (GFR $<60 \mathrm{~mL} / \mathrm{min}$ ) adhered to the respective $\mathrm{SmPC}$ either because the drug was used in patients with renal impairment (although its use was contraindicated in this setting) or because the dosage was not adjusted as recommended. Moreover, the authors recognized that the official product labeling for cisplatin, ifosfamide, and etoposide varies between products and countries. There was also little agreement between the approved product labeling and literature recommendations regarding dose adjustments in cancer patients with renal dysfunction.

Unfortunately, this most recent report is not unique. Several papers similarly concluded that there was poor consistency in information from sources for the renal dosing of commonly used drugs (Khanal et al. 2014). The poor consistency in dosing recommendations may reflect insufficient clinical evidence. Indeed, most cancer drugs were approved with limited information on the pharmacokinetics and/or pharmacodynamics of the drugs in patients with renal dysfunction. Traditionally, the majority of phase I studies in oncology 
excluded patients with moderate to severe renal dysfunction, primarily due to safety concerns. This is true for cisplatin, ifosfamide, and etoposide, which were approved in the 1970s and 1980s, as well as other oncology drugs, most of which were approved before 2000. Of these, only a few drugs, such as etoposide, have been investigated post marketing in renal impairment studies and, therefore, have information on dose adjustments in renal dysfunction available in their SmPC. Due to lack of information about the appropriate dose, anticancer medications, such as cisplatin and ifosfamide, are formally contraindicated for patients with renal dysfunction in some countries. To withhold cytostatic drug treatment from these patients is not consistent with clinical guidelines and can decrease the chances of cure or progression-free survival if no equi-effective alternative treatment is available. In addition to the SmPCs, guidelines and textbooks are available on how to adjust anticancer drug dosages according to renal function (Aronoff et al. 2007). They rely in many cases on lower levels of evidence, such as case reports or experimental studies, or simply summarize expert opinions. Frequently utilized reference sources that were established many years ago require revisions and updates. Thus, guideline and textbook recommendations also have limitations.

The poor consistency in the official product labeling between products and countries, as reported by Grafe et al. 2018, may be surprising because instruments for the harmonization of product labeling, at least across the European Union (EU), are in place. An example is a recent change in the drug labeling of metformin, which varied between products and EU countries and was no longer consistent with clinical guidelines. Following a request from the Dutch medicines agency, the European Medicines Agency (EMA) updated and harmonized the product information for the EU in 2016 (Committee for Medicinal Products for Human Use 2016). This included a revision of the contraindications and information regarding doses, monitoring, and precautions in patients with reduced kidney function. However, a procedure, as recently conducted for metformin, can only be successful if clinical data is available and the level of evidence is sufficient to support a harmonized position.

Approval authorities have recognized that it is imperative that new drug applications should include information on the impact of renal clearance and whether posology adjustments are required for patients with renal impairment. To this end, the EMA released the "Guideline on the evaluation of the pharmacokinetics of medicinal products in patients with decreased renal function," effective from July 2016 (European Medicines Agency 2015). Already in 1998, the US Food and Drug Administration (FDA) issued its first guidance on renal impairment studies, which was subsequently revised in 2010 (US Food and Drug Administration 2010). The EMA and FDA guideline documents have accelerated the availability of safety data for novel anticancer therapies in renal dysfunction patients. Specific pharmacokinetics and safety studies for many of the newly approved drugs were performed in a setting of renal impairment to provide the basis for harmonized dosing recommendations, especially for patients with mild or moderate renal insufficiency. Although the number, quality, and consistency of dosing recommendations in SmPCs for newly approved drugs have increased, there is room for improvement. Guidance for dose adjustment in patients with end-stage kidney disease or hemodialysis, for example, is missing for many of the new drug approvals. Moreover, a recent survey of renal impairment studies on new oncology drug approvals by the FDA from 2010 to 2015 found that only $28 \%$ of the drugs had a dedicated renal impairment study included in their initial new drug application (Xiao et al. 2017).

Population aging can increase the number of patients with chronic kidney disease. Therefore, accessible and understandable information about the use of anticancer drugs in this group of patients becomes increasingly important. Reliable information on posology adjustments is also the basis for integration into emerging health information technologies, such as electronic clinical decision support systems, which are expected to improve drug prescribing quality, particularly in patients with kidney disease. In 2010, the KDIGO conference brought together 50 experts, including representatives from the EMA and FDA, to propose recommendations for research and regulatory authorities, which remain valid: rigorously conducted renal impairment studies are needed and drug labeling should be improved (Matzke et al. 2011). It might be time to take action.

\section{References}

Aronoff GR, Bennett WM, Berns JS et al (2007) Drug prescribing in renal failure: dosing guidelines for adults and children, 5th edn. American College of Physicians, Philadelphia

Committee for Medicinal Products for Human Use (2016) Assessment report: Referral under Article 31 of Directive 2001/83/EC, metformin containing medicinal products

European Medicines Agency (2015) Guideline on the evaluation of the pharmacokinetics of medicinal products in patients with decreased renal function

GBD (2016) Disease and Injury Incidence and Prevalence Collaborators (2017) Global, regional, and national incidence, prevalence, and years lived with disability for 328 diseases and injuries for 195 countries, 1990-2016: a systematic analysis for the Global Burden of Disease Study 2016. Lancet 390:1211-1259. https://doi.org/10. 1016/S0140-6736(17)32154-2

Grafe C, Semrau S, Hein A, Beckmann MW, Mackensen A, Dörje F, Fromm MF (2018) Dose adjustment of cisplatin, etoposide and ifosfamide according to kidney function: a retrospective analysis and implications for medication safety. Naunyn Schmiedebergs Arch Pharmaco. https://doi.org/10.1007/s00210-017-1456-2

Hill NR, Fatoba ST, Oke JL, Hirst JA, O'Callaghan CA, Lasserson DS, Hobbs FD (2016) Global prevalence of chronic kidney disease - a 
systematic review and meta-analysis. PLoS One 11(7):e0158765. https://doi.org/10.1371/journal.pone.0158765

Khanal A, Castelino RL, Peterson GM, Jose MD (2014) Dose adjustment guidelines for medications in patients with renal impairment: how consistent are drug information sources? Intern Med J 44(1):77-85. https://doi.org/10.1111/imj.12291

Launay-Vacher V, Janus N, Deray G (2016) Renal insufficiency and cancer treatments. ESMO Open 1(4):e000091. https://doi.org/10. 1136/esmoopen-2016-000091

Matzke GR, Aronoff GR, Atkinson AJ Jr, Bennett WM, Decker BS, Eckardt KU, Golper T, Grabe DW, Kasiske B, Keller F, Kielstein JT, Mehta R, Mueller BA, Pasko DA, Schaefer F, Sica DA, Inker LA, Umans JG, Murray P (2011) Drug dosing consideration in patients with acute and chronic kidney disease-a clinical update from
Kidney Disease: Improving Global Outcomes (KDIGO). Kidney Int 80(11):1122-1137. https://doi.org/10.1038/ki.2011.322

US Food and Drug Administration (2010) Guidance for industry: pharmacokinetics in patients with impaired renal function - study design, data analysis, and impact on dosing and labeling

Velenosi TJ, Urquhart BL (2014) Pharmacokinetic considerations in chronic kidney disease and patients requiring dialysis. Expert Opin Drug Metab Toxicol 10(8):1131-1143. https://doi.org/10.1517/ 17425255.2014.931371

Xiao JJ, Chen JS, Lum BL, Graham RA (2017) A survey of renal impairment pharmacokinetic studies for new oncology drug approvals in the USA from 2010 to early 2015: a focus on development strategies and future directions. Anti-Cancer Drugs 28(7):677-701. https://doi.org/10.1097/CAD.0000000000000513 